\title{
INVARIANT ALGEBRAIC SURFACES AND HOPF-BIFURCATION OF A FINANCE MODEL
}

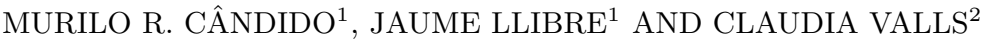

ABstRACT. Recently there are several works studying the finance model

$$
\dot{x}=z+x(y-a), \quad \dot{y}=1-b y-x^{2}, \quad \dot{z}=-x-c z
$$

where $a, b$ and $c$ are positive parameters. The first objective of this paper is to show that this model exhibits one small amplitude periodic solution emerging from a Hopf bifurcation at the equilibrium point $(0,1 / b, 0)$ and in the second one we show that this system does not have invariant algebraic surfaces for any value of the parameters.

\section{IntRodUCTION AND STATEMENT OF THE MAIN RESULTS}

We consider the following polynomial differential system in $\mathbb{R}^{3}$

$$
\begin{aligned}
& \dot{x}=z+x(y-a), \\
& \dot{y}=1-b y-x^{2}, \\
& \dot{z}=-x-c z,
\end{aligned}
$$

where $a, b$ and $c$ are real positive parameters and the dot denotes derivative with respect to the time $t$. This model has been intensively investigated (see, for instance, $[1,2,4,5,8,10]$. It describes the time variation of these state variables: the interest rate $x$, the investment demand $y$ and the price index $z$. Here $a$ is the saving amount, $b$ is the cost per investment and $c$ is the elasticity of demand of commercial market. Changes in $x$ come from an excess of investment over savings and the structural adjustment from good prices. Changes in $y$ are in proportion to the rate of investment and to an inversion with the cost of investment and interest rates. Finally, changes in $z$ are controlled by inflation rates.

The first objective of the present paper is to study the Hopf bifurcation which exhibits the polynomial differential system (1). We recall that a Hopf bifurcation in $\mathbb{R}^{3}$ takes place in an equilibrium point with eigenvalues of the form $\pm \omega i$ and $\lambda$, with $\omega, \lambda \in \mathbb{R}$. The Hopf bifurcation theory is well understood (see [6]). Our analysis of the Hopf bifurcation will be directly

2010 Mathematics Subject Classification. Primary 34A05. Secondary 34C05, 37C10. Key words and phrases. Darboux integrability, Hopf bifurcation, averaging theory, invariant algebraic surface, Lyapunov constant. 
computing the first Liapunov coefficient and also using averaging theory to overcome the cases in which the first Liapunov coefficient is zero.

System (1) has at most the following tree equilibrium points

$$
\begin{aligned}
P & =\left(0, \frac{1}{b}, 0\right), \\
P_{ \pm} & =\left(\mp \sqrt{\frac{c-b(a c+1)}{c}}, a+\frac{1}{c}, \pm \sqrt{\frac{c-b(a c+1)}{c^{3}}}\right), \quad \text { if } c-b(a c+1)>0 .
\end{aligned}
$$

We can check that $P$ is a Hopf point of system (1) with bifurcation parameter $\bar{a}=(1-b c) / b$ and has eigenvalues $b$ and $\pm i \bar{c}$, where $\bar{c}=\sqrt{1-c^{2}}$ with $c<1$. The proof is immediate by computing directly the eigenvalues at each equilibrium point. We expect to have small-amplitude limit cycles branching from this fixed point. This is the main result of this paper.

Theorem 1. Let

$$
(a, b, c) \in \mathbb{R}^{3} \quad \text { with } \quad \bar{a}=\frac{1-b c}{b}>0 \quad \text { and } \quad 0<c<1 .
$$

For a sufficiently close to the bifurcation value $\bar{a}$, the following statements hold. The first Liapunov constant is

$$
l_{1}=-\frac{(3 b+4 c)(b-2 c)+8}{8 b \bar{c}\left(b^{2}+4 \bar{c}^{2}\right)} \neq 0 .
$$

(i) If $l_{1}>0$, system (1) has a supercritical Hopf bifurcation at the equilibrium point $P$, i.e., the point $P$ is a weak focus of system (1) restricted to the central manifold of $P$ and the limit cycle that emerges from $P$ is stable.

(ii) If $l_{1}<0$, system (1) has a subcritical Hopf bifurcation at the equilibrium point $P$, i.e., the point $P$ is a weak focus of system (1) restricted to the central manifold of $P$ and the limit cycle that emerges from $P$ is unstable.

Theorem 1 is proved in section 2. To compute the first Liapunov constant we use a result in [6]. We note that there are three situations in which the first Liapunov coefficient does not provide results, i.e. when

(a) $b=\frac{2}{5} \sqrt{\frac{2}{3}}$ and $c=\frac{2}{5} \sqrt{6}$,

(b) $b=\frac{c}{3}-\frac{1}{3} \sqrt{25 c^{2}-24}$ and $\frac{2 \sqrt{6}}{5}<c<1$,

(c) $b=\frac{c}{3}+\frac{1}{3} \sqrt{25 c^{2}-24}$ and $\frac{2 \sqrt{6}}{5}<c<1$.

In cases $(a)-(c)$ higher order Liapunov's coefficient would be necessary to describe the existence of periodic solutions emerging from the singular point 
$P$. In the next result we show that the averaging method can be used to overcome this difficulty.

Theorem 2. Consider the differential system (1) with $0<c<1, a_{2} \neq 0$ and $a=\bar{a}+\varepsilon^{2} a_{2}$. Assume that

(2) $a_{2}\left(4 \bar{c}\left(1-e^{-\frac{2 \pi b}{\bar{c}}}\right)\left(b^{2}+2 \bar{c}^{2}\right)\left(b^{2}-b c+2 \bar{c}^{2}\right)-8 \pi l_{1}\left(b^{3}+4 b \bar{c}^{2}\right)^{2}\right)>0$.

Then for $|\varepsilon|>0$ sufficiently small, system (1) has a periodic solution bifurcating from the equilibrium point $P$.

We note that for the values (a)-(c) condition (2) holds and consequently Theorem 2 shows the existence of a Hopf bifurcation in the cases not covered by Theorem 1 . In fact for condition (a) the inequality (2) becomes

$$
\frac{112 a_{2}}{5625}\left(e^{-4 \sqrt{\frac{2}{3}} \pi}-1\right)>0 .
$$

Moreover, for condition (b) the inequality (2) writes $a_{2} p(c)>0$ with

$$
\begin{aligned}
p(c)= & \frac{8}{81} \sqrt{1-c^{2}}\left(5 c^{2}-6-c \sqrt{25 c^{2}-24}\right) \\
& \left(c\left(4 c+\sqrt{25 c^{2}-24}\right)-3\right) e^{-\frac{2 \pi\left(\sqrt{25 c^{2}-24}+c\right)}{3 \sqrt{1-c^{2}}}} .
\end{aligned}
$$

We notice that $p(c) \neq 0$ for all $\frac{2 \sqrt{6}}{5}<c<1$. Mainly $p(c)=0$ if and only if $c=-1$, thus in the cases (a) and (b) we can always choose a convenient $a_{2} \in \mathbb{R}$ such that condition (2) holds. By a similar argument the same holds for condition (c).

Theorem 2 is proved in section 3.

The second objective of this paper is to study the existence of invariant algebraic surfaces for system (1). We recall that the study of invariant objects is one of the main tools in dynamical systems. To introduce the definition of invariant algebraic surface, let $f(x, y, z)$ be a real polynomial in the variables $x, y, z$. We say that $f$ is Darboux polynomial of system (1) if it satisfies

$$
\frac{\partial f}{\partial x}(z+x(y-a) y)+\frac{\partial f}{\partial y}\left(1-b y-x^{2}\right)-\frac{\partial f}{\partial z}(x+c z)=K f
$$

for some real polynomial $K(x, y, z)$ of degree at most one, which is called the cofactor of $f$. If $f(x, y, z)$ is Darboux polynomial and then the surface $f=0$ is invariant and it is called an invariant algebraic surface. We note that if a solution of (1) has a point on an invariant algebraic surface, then the whole solution is contained in it.

The second main theorem is the following.

Theorem 3. System (1) has no invariant algebraic curves. 
The proof of Theorem 3 is given in section 4 . We have also included some appendix with the results that we shall use to prove Theorems 1 and 2 .

\section{Proof of Theorem 1}

The proof will be done using Lemma 6 . Recall that the bifurcation coefficient is $\bar{a}=(1-b c) / b$, and the eigenvalues are $\pm i \bar{c}= \pm i \sqrt{1-c^{2}}$ and $b$.

The characteristic polynomial of the Jacobian matrix of system (1) at $P$ is

$$
-\lambda^{3}+\frac{\lambda^{2}(1-b(a+b+c))}{b}+\lambda\left(-a(b+c)-b c+\frac{c}{b}\right)-b(a c+1)+c=0 .
$$

Let $\lambda(a)$ be a real root of the characteristic polynomial. It depends continuously on $a$ and we can write

(3) $\frac{d \lambda}{d a}(a)=-\frac{b(\lambda(a)+b)(\lambda(a)+c)}{2 \lambda(a)(b(a+b+c)-1)+a b(b+c)+3 b \lambda(a)^{2}+\left(b^{2}-1\right) c}$.

Let $\lambda(\bar{a})=i \bar{c}$. Taking $a=\bar{a}$ in (3) we have

$$
\operatorname{Re}\left(\frac{d \lambda}{d a}(\bar{a})\right)=\frac{(\bar{c}-c)(\bar{c}-b)}{2 \bar{c}(b-2 \bar{c})} \neq 0 .
$$

Thus, in order to apply Lemma 6 accordingly with the arguments in Section 5.1 , we need to calculate

$$
A=\left(\begin{array}{ccc}
c & 0 & 1 \\
0 & -b & 0 \\
-1 & 0 & -c
\end{array}\right),
$$

and the multilinear functions $B(\mathbf{x}, \mathbf{y})=\left(x_{2} y_{1},+x_{1} y_{2}, 2 x_{1} x_{2}, 0\right)$ and $C(\mathbf{x}, \mathbf{y}, \mathbf{z})=$ $(0,0,0)$. We also obtain the eigenvectors

$$
p=\left(-\frac{i}{\bar{c}}, 0, \frac{\bar{c}-i}{\bar{c}}\right), \quad q=\frac{1}{2}(-c-i \bar{c}, 0,1) .
$$

In this case the first Liapunov coefficient (see (13))

$$
l_{1}=\frac{1}{2 \omega} \operatorname{Re}\left(-2 \bar{p} \cdot B\left(q, A^{-1} \cdot B(q, \bar{q})\right)+\bar{p} B\left(\bar{q},\left(2 \omega i I_{n}-A\right)^{-1} B(q, q)\right)\right)
$$

is equal to

$$
l_{1}=-\frac{(3 b+4 c)(b-2 c)+8}{8 b \bar{c}\left(b^{2}+4 \bar{c}^{2}\right)} .
$$

So the theorem follows directly from Lemma 6 . 


\section{Proof of Theorem 2}

We first do a reescaling of the system by doing $(x, y, z)=\varepsilon(\bar{x}, \bar{y}, \bar{z})$. Then we do the linear change of coordinates

$$
(\bar{x}, \bar{y}, \bar{z})=(X, Z-\bar{c} Y-c Z)
$$

obtaining the system

$$
\begin{aligned}
\dot{X} & =-\bar{c} Y+\varepsilon X(Z)-\varepsilon^{2} a_{2} X, \\
\dot{Y} & =\bar{c} X-\varepsilon \frac{c X Z}{\bar{c}}+\varepsilon^{2} \frac{a_{2} c X}{\bar{c}}, \\
\dot{Z} & =-b Z-\varepsilon X^{2} .
\end{aligned}
$$

Then we consider the cylindrical coordinates $X=r \cos \theta, Y=r \sin \theta, Z=z$ in system (4) and we take $\theta$ as the new independent variable. In this way we obtain a differential system of the form

$$
\left(r^{\prime}, z^{\prime}\right)=F_{0}(r, z)+\varepsilon F_{1}(r, z, \theta)+\varepsilon^{2} F_{2}(r, z, \theta)+\mathcal{O}\left(\varepsilon^{3}\right),
$$

where the prime denotes the derivative with respect to $\theta$, and

$$
F_{0}(r, z)=\left(0,-\frac{b z}{\bar{c}}\right), \quad F_{i}(r, z, \theta)=\left(F_{i}^{1}(r, z, \theta), F_{i}^{2}(r, z, \theta)\right), i=1,2
$$

with

$$
\begin{aligned}
F_{1}^{1}(r, z, \theta)= & r z \cos \theta\left(\frac{\cos \theta}{\bar{c}}-\frac{c \sin \theta}{\bar{c}^{2}}\right), \\
F_{1}^{2}(r, z, \theta)= & -\cos \theta\left(b c z^{2}+\bar{c}^{2} r^{2}\right) \frac{\cos \theta}{\bar{c}^{3 / 2}}-b \bar{c} z^{2} \sin \theta, \\
F_{2}^{1}(r, z, \theta)= & \frac{r \cos \theta}{\bar{c}^{5}}\left(a_{2} c \bar{c}^{3} \sin \theta+\bar{c}^{2} \cos \theta\left(-a_{2} \bar{c}^{2}+c z^{2} \cos (2 \theta)\right)\right. \\
+ & \left.\left(1-2 c^{2}\right) \bar{c} z^{2} \sin \theta \cos ^{2} \theta\right), \\
F_{2}^{2}(r, z, \theta)= & \frac{z \cos \theta}{2 \bar{c}^{5}}\left(2 a_{2} b \bar{c}^{3} \sin \theta+b \bar{c}^{2} \cos \theta\left(2 a_{2} c+z^{2} \cos (2 \theta)-z^{2}\right)\right. \\
& \left.+2 c \cos ^{3} \theta\left(-\bar{c}^{2} r^{2}-b c z^{2}\right)+2 \bar{c} \sin \theta \cos ^{2} \theta\left(-\bar{c}^{2} r^{2}-2 b c z^{2}\right)\right) .
\end{aligned}
$$

Now we use (16) to obtain the following averaged functions of system (5):

$$
\mathbf{g}_{0}(r, z)=\left(0,\left(1-e^{\frac{2 \pi b}{\bar{c}}}\right) z\right), \quad \mathbf{g}_{i}(r, z)=\left(\mathbf{g}_{i}^{1}(r, z), \mathbf{g}_{i}^{2}(r, z)\right), i=1,2
$$


with

$$
\begin{aligned}
\mathbf{g}_{1}^{1}(r, z)= & \left(1-e^{-\frac{2 \pi b}{\bar{c}}}\right) \frac{r z((b+c)(b-2 c)+2)}{2 \pi b\left(b^{2}+4 \bar{c}^{2}\right)}, \\
\mathbf{g}_{1}^{2}(r, z)= & \left(e^{-\frac{4 \pi b}{\bar{c}}}-e^{-\frac{2 \pi b}{\bar{c}}}\right) \frac{1}{2 \pi b \bar{c}^{2}\left(b^{2}+4 \bar{c}^{2}\right)}\left(-\bar{c}^{2} r^{2}\left(b^{2}+2 \bar{c}^{2}\right) e^{\frac{2 \pi b}{\bar{c}}}\right. \\
& \left.-b z^{2}\left(b^{2} c+2 c \bar{c}^{2}+b \bar{c}\right)\right), \\
\mathbf{g}_{2}^{1}(r, z)= & r\left(\left(1-e^{-\frac{2 \pi b}{\bar{c}}}\right) \frac{z((b+c)(b-2 c)+2)}{b\left(b^{2}+4 \bar{c}^{2}\right)}-\frac{\pi a_{2}}{\bar{c}}\right), \\
\mathbf{g}_{2}^{2}(r, z)= & \frac{e^{-\frac{4 \pi b}{\bar{c}}}}{b \bar{c}^{3}\left(b^{2}+4 \bar{c}^{2}\right)}\left(e ^ { \frac { 2 \pi b } { \overline { c } } } \left(b z\left(\pi a_{2} b c\left(b^{2}+4 \bar{c}^{2}\right)-\bar{c} z\left(b^{2} c+b c+2 c \bar{c}^{2}\right)\right)\right.\right. \\
+ & \left.\left.\bar{c}^{3} r^{2}\left(b^{2}+2 \bar{c}^{2}\right)\right)+\bar{c}^{3} r^{2}\left(-b^{2}+\bar{c}^{2}\right) e^{\frac{4 \pi b}{\bar{c}}}+b \bar{c} z^{2}\left(b^{2} c+(2 c+b) \bar{c}^{2}\right)\right) .
\end{aligned}
$$

The function $\mathbf{g}_{0}(\mathbf{z})$ vanishes on the the graph $\mathcal{Z}=\{(r, 0): r>0\}$. Hence, we apply Theorem 7 to system (5) with

$$
\Delta_{\alpha}=1-e^{\frac{2 \pi b}{\bar{c}}} \neq 0 .
$$

Computing the bifurcation functions we get

$$
\begin{aligned}
f_{1}(r) & =0, \\
f_{2}(r) & =-\frac{\pi a_{2} r}{\bar{c}}+\frac{r^{3}}{4 b^{2} \bar{c}\left(b^{2}-4 c^{2}+4\right)^{2}}\left(\left(4 \bar{c}\left(b^{2}+2 \bar{c}^{2}\right)\left(b^{2}-b c+2 \bar{c}^{2}\right)\right.\right. \\
& \left.-\pi b\left(b^{2}+4 \bar{c}^{2}\right)\left(3 b^{2}-2 b c+8 \bar{c}^{2}\right)\right)-4 \bar{c}\left(b^{2}+2 \bar{c}^{2}\right) \\
& \left.e^{-\frac{2 \pi b}{\bar{c}}}\left(b^{2}-b c+2 \bar{c}^{2}\right)\right) .
\end{aligned}
$$

Let

$$
\bar{r}=2 b\left(b^{2}+4 \bar{c}^{2}\right) e^{\frac{\pi b}{\bar{c}}} \sqrt{\frac{a_{2} \pi}{N}}
$$

where

$$
\begin{aligned}
& N=4 \bar{c}\left(b^{2}-2 c^{2}+2\right)((b+c)(b-2 c)+2)\left(e^{\frac{2 \pi b}{\bar{c}}}-1\right)-\pi b\left(b^{2}-4 c^{2}+4\right) \\
& \quad((3 b+4 c)(b-2 c)+8) e^{\frac{2 \pi b}{\bar{c}}} .
\end{aligned}
$$

Note that if $a_{2} N>0$, then $f_{2}(r)=0$ has the positive solution $\bar{r}$. Furthermore

$$
f_{2}^{\prime}(\bar{r})=\frac{2 \pi a_{2}}{\bar{c}} \neq 0,
$$

and consequently, by Theorem 7(b), system (5) has a periodic solution bifurcating from the origin. The result follows going back through the changes of coordinates. 


\section{Proof of Theorem 3}

Note that system (1) is invariant under the automorphism $\tau: \mathbb{R} \rightarrow \mathbb{R}$ of the form

$$
\tau(x)=-x, \quad \tau(y)=y, \quad \tau(z)=-z .
$$

Moreover we have the following well-known proposition (for a proof see [7]).

Proposition 4. Let $f=f(x, y, z)$ be an invariant algebraic surface of system (1) with cofactor $K$. Then $g=f \cdot \tau(f)$ is another invariant algebraic surface of system (1) invariant by $\tau$ with cofactor $K+\tau(K)$.

We first prove Theorem 3 for invariant algebraic surfaces that are invariant by $\tau$. After that, we prove it for general invariant surfaces via Proposition 4. Hence, we shall first prove the following theorem.

Theorem 5. System (1) has no invariant algebraic surfaces that are invariant by $\tau$.

To prove Theorem 5 we recall that a polynomial $g(X)$ with $X \in \mathbb{R}^{n}$ is said to be weight homogeneous if there exists $s=\left(s_{1}, \ldots, s_{n}\right) \in \mathbb{N}^{n}$ and $m \in \mathbb{N}$ such that for all $\mu \in \mathbb{R} \backslash\{0\}, g\left(\mu^{s} X\right)=g\left(\mu^{s_{1}} x_{1}, \ldots, \mu^{s_{n}} x_{n}\right)=\mu^{m} g(X)$, where $\mathbb{N}$ the set of positive integers. We shall refer $s$ to be the weight of $g$, $m$ the weight degree, $X \mapsto \mu^{s} X$ the weight change of the variables.

Proof of Theorem 5. To prove Theorem 5 we do the change of variables and the time-reversion

$$
X=x, \quad Y=y-a, \quad Z=z, \quad T=-t .
$$

Then system (1) becomes

$$
\begin{aligned}
& x^{\prime}=-z-x y, \\
& y^{\prime}=-\tilde{a}+b y+x^{2}, \\
& z^{\prime}=x+c z,
\end{aligned}
$$

where $\tilde{a}=1-a b$, the prime means derivative with respect the new time $T$ and we have renamed the new variables $(X, Y, Z)$ again as $(x, y, z)$.

Note that in order to prove Theorem 5 for system (1) it is sufficient to prove it for system (6).

Now we do the change of variables

$$
x=\mu^{-1} X, \quad y=\mu^{-1} Y, \quad z=Z, \quad T=\mu \tau .
$$

Under this change of variables system (6) becomes

$$
\begin{aligned}
& X^{\prime}=-X Y-\mu^{2} Z, \\
& Y^{\prime}=X^{2}+\mu b Y-\mu^{2} \tilde{a}, \\
& Z^{\prime}=X+\mu c Z,
\end{aligned}
$$


where the prime denotes the derivative of the variables with respect to $\tau$.

Assume that $f(x, y, z)$ is a Darboux polynomial of system (6) invariant by $\tau$ with cofactor $K(x, y, z)$. Without loss of generality we can assume that the cofactor is invariant by $\tau$ (see Proposition 4) and so $K(x, y, z)=k_{0}+k_{2} y$. Set

$$
F(X, Y, Z)=\mu^{\ell} f\left(\mu^{-1} X, \mu^{-1} Y, Z\right)
$$

and

$$
K(X, Y, Z)=\mu k\left(\mu^{-1} X, \mu^{-1} Y, Z\right),
$$

where $\ell$ is the highest weight degree in the weight homogeneous components of $f$ in $x, y$ and $z$ with the weight $(1,1,0)$. Assume that $F=\sum_{i=0}^{m} \mu^{i} F_{i}$ where $F_{i}$ is a weight homogeneous polynomial in $X, Y$ and $Z$ with the weight degree $\ell-i$ for $i=0,1, \ldots, m$ and $\ell \geq m$. From the definition of a Darboux polynomial we have

$$
\begin{aligned}
& -\left(x y+\mu^{2} z\right) \sum_{i=0}^{m} \mu^{i} \frac{\partial F_{i}}{\partial x}+\left(x^{2}+\mu b y-\mu^{2} \tilde{a}\right) \sum_{i=0}^{m} \frac{\partial F_{i}}{\partial y} \\
& +(x+\mu c z) \sum_{i=0}^{m} \mu^{i} \frac{\partial F_{i}}{\partial z}=\left(k_{2} y+\mu k_{0}\right) \sum_{i=0}^{m} \mu^{i} F_{i},
\end{aligned}
$$

where we still use $x, y, z$ instead of $X, Y$ and $Z$. Equating the terms with $\mu^{i}$ for $i=0,1, \ldots, m+2$ we get

$$
\begin{aligned}
& L\left[F_{0}\right]=k_{2} y F_{0}, \\
& L\left[F_{1}\right]=k_{2} y F_{1}+k_{0} F_{0}-b y \frac{\partial F_{0}}{\partial y}+c z \frac{\partial F_{0}}{\partial z} \\
& L\left[F_{j}\right]=k_{2} y F_{j}+k_{0} F_{j-1}-b y \frac{\partial F_{j-1}}{\partial y}-c z \frac{\partial F_{j-1}}{\partial z}+z \frac{\partial F_{j-2}}{\partial x}+\tilde{a} \frac{\partial F_{j-2}}{\partial y},
\end{aligned}
$$

for $j=2,3, \ldots, m+2$ where $F_{j}=0$ for $j>m$ and $L$ is the linear partial differential operator of the form

$$
L=-x y \frac{\partial}{\partial x}+x^{2} \frac{\partial}{\partial y}+x \frac{\partial}{\partial z} .
$$

The system associated to the linear partial differential operator $L$ is

$$
x^{\prime}=-x y, \quad y^{\prime}=x^{2}, \quad z^{\prime}=x .
$$

This system has the general solutions

$$
x^{2}+y^{2}=d_{1}, \quad z-\arctan \left(\frac{x}{y}\right)=d_{2}
$$

where $d_{1}$ and $d_{2}$ are constants of integration. According to this, we do the change of variables

$$
u=x^{2}+y^{2}, \quad v=z-\arctan \left(\frac{y}{x}\right), \quad w=y,
$$


whose inverse transformation is

$$
x= \pm \sqrt{u-w^{2}}, \quad z=v+\arctan \left(\frac{y}{ \pm \sqrt{u-w^{2}}}\right), \quad y=w .
$$

In the following for simplicity we only consider the case $x=\sqrt{u-w^{2}}$, $z=v+\arctan \left(\frac{y}{\sqrt{u-w^{2}}}\right)$ and $y=w$. In this paper we always use the notation $\bar{f}$ to denote a function $f(x, y, z)$ written in the variables $u, v, w$.

Under the changes of variables (8) the first equation of (7) becomes the following ordinary differential equation (for fixed $u, v$ ):

$$
\left(u-w^{2}\right) \frac{d \bar{F}_{0}}{d w}=k_{2} w \bar{F}_{0}
$$

This equation has the general solution

$$
\bar{F}_{0}=\left(u-w^{2}\right)^{k_{2} / 2} \bar{G}_{0}(u, v),
$$

where $\bar{G}_{0}$ is an arbitrary smooth function in the variables $u$ and $v$. So, using (9) we obtain

$$
F_{0}(x, y, z)=\bar{F}_{0}(u, v, w)=x^{k_{2} / 2} \bar{G}_{0}\left(x^{2}+y^{2}, z-\arctan \left(\frac{y}{x}\right)\right) .
$$

In order that $F_{0}$ be a weight homogeneous polynomial, we must have that $G_{0}$ can only depend on $x^{2}+y^{2}$, and so $\bar{G}_{0}$ a polynomial in $u$. Since $F_{0}$ must be a polynomial and also must be invariant by $\tau$, we must have that $k_{2} / 2$ is even, i.e., $k_{2}=4 \tilde{k}_{2}$. Moreover, taking into account that the weight degrees of $u$ and $v$ in $x, y, z$ are 1,1 and 0 , respectively. But $\bar{F}_{0}$ does not depend on $v$, we get that $F_{0}$ should have weight degree $\ell=2 n$. So, $\ell$ is even and

$$
F_{0}=\alpha_{0} x^{2 \tilde{k}_{2}}\left(x^{2}+y^{2}\right)^{n-\tilde{k}_{2}}, \quad \text { where } \alpha_{0} \in \mathbb{R} \backslash\{0\} .
$$

Now we compute $F_{1}$. It follows from $(7)$ that

$$
\begin{aligned}
L\left[F_{1}\right] & =4 \tilde{k}_{2} y F_{1}+k_{0} F_{0}-b y \frac{\partial F_{0}}{\partial y}-c z \frac{\partial F_{0}}{\partial z} \\
& =4 \tilde{k}_{2} y F_{1}+\alpha_{0} x^{2 \tilde{k}_{2}}\left(x^{2}+y^{2}\right)^{n-\tilde{k}_{2}-1}\left(k_{0}\left(x^{2}+y^{2}\right)-2 b\left(\tilde{k}_{2}-n\right) y^{2}\right) .
\end{aligned}
$$

Using the transformations (8) and (9) and working in a similar way to solve $\bar{F}_{0}$, we get the following ordinary differential equation when $u$ and $v$ are fixed:

$$
\left(u-w^{2}\right) \frac{d \bar{F}_{1}}{d w}=2 \tilde{k}_{2} w \bar{F}_{1}+\alpha_{0} u^{n-\tilde{k}_{2}-1}\left(u-w^{2}\right)^{\tilde{k}_{2}}\left(k_{0} u-2 b\left(\tilde{k}_{2}-n\right) w^{2}\right) .
$$

Solving this linear differential equation we get

$$
\begin{aligned}
\bar{F}_{1}= & \left(u-w^{2}\right)^{\tilde{k}_{2}} G_{1}(u, v)+\alpha_{0}\left(u-w^{2}\right)^{\tilde{k}_{2}} u^{n-\tilde{k}_{2}-1}\left(\left(2 b\left(n-\tilde{k}_{2}\right) w\right.\right. \\
& \left.-\left(k_{0}-2 b\left(n-\tilde{k}_{2}\right)\right) \sqrt{u} \arctan \left(\frac{w}{\sqrt{u}}\right)\right)
\end{aligned}
$$


where $G_{1}$ is a smooth function in the variables $u, v$. Hence,

$$
\begin{aligned}
F_{1}= & \alpha_{1} x^{2 \tilde{k}_{2}} G_{1}\left(x^{2}+y^{2}, z-\arctan \left(\frac{y}{x}\right)\right)+\alpha_{0} x^{2 \tilde{k}_{2}}\left(x^{2}+y^{2}\right)^{n-\tilde{k}_{2}-1} \\
& \times\left(2 b\left(\tilde{k}_{2}-n\right) y-\left(k_{0}-2 b\left(n-\tilde{k}_{2}\right)\right) \sqrt{x^{2}+y^{2}} \arctan \left(\frac{y}{\sqrt{x^{2}+y^{2}}}\right)\right) .
\end{aligned}
$$

In order that $F_{1}$ be a weight homogeneous polynomial of weight degree $2 n-1$ we must have $G_{1}=0$ and $k_{0}=2 b\left(n-\tilde{k}_{2}\right)$. Doing that we have

$$
F_{1}=2 b \alpha_{0}\left(\bar{k}_{2}-n\right) x^{2 \tilde{k}_{2}}\left(x^{2}+y^{2}\right)^{n-\tilde{k}_{2}-1} y .
$$

Now substituting $F_{0}$ and $F_{1}$ given in (10) and (11) into equation (7) we get

$$
\begin{aligned}
L\left[F_{2}\right]= & 2 \tilde{k}_{2} y F_{2}-2 \alpha_{0} x^{2 \tilde{k}_{2}-1}\left(x^{2}+y^{2}\right)^{n-\tilde{k}_{2}-2}\left(( \tilde { k } _ { 2 } - n ) x y \left(\left(\tilde{a}-b^{2}\right.\right.\right. \\
& \left.\left.\left.-2 b^{2}\left(\tilde{k}_{2}-n\right)\right) x^{2}+\left(\tilde{a}+b^{2}\right) y^{2}\right)-\left(x^{2}+y^{2}\right)\left(n x^{2}+\tilde{k}_{2} y^{2}\right) z\right) .
\end{aligned}
$$

Using the transformations (8) and (9) and working in a similar way to solve $\bar{F}_{1}$, we get the following ordinary differential equation when $u$ and $v$ are fixed:

$$
\begin{aligned}
\left(u-w^{2}\right) \frac{d \bar{F}_{2}}{d w}= & 2 \tilde{k}_{2} w \bar{F}_{2}+2 \alpha_{0} u^{n-\tilde{k}_{2}-2}\left(u-w^{2}\right)^{\tilde{k}_{2}-1 / 2}\left(b^{2}\left(\tilde{k}_{2}-n\right) w \sqrt{u-w^{2}}\right. \\
& \left(\left(\tilde{a}+b^{2}\right) w^{2}+\left(\tilde{a}-b^{2}-2 b^{2}\left(\tilde{k}_{2}-n\right)\right)\left(u-w^{2}\right)\right) \\
& \left.-u\left(n u+\left(\tilde{k}_{2}-n\right) w^{2}\right)\left(v+\arctan \left(\frac{w}{\sqrt{u-w^{2}}}\right)\right)\right) .
\end{aligned}
$$

Solving this linear differential equation we get

$$
\begin{aligned}
\bar{F}_{2}= & \left(u-w^{2}\right)^{\tilde{k}_{2}} G_{2}(u, v)-\alpha_{0} u^{n-k_{2}-2}\left(u-w^{2}\right)^{\tilde{k}_{2}}\left(2 \left(\left(k_{2}-n\right) u v\right.\right. \\
& \left.-\frac{k_{2} u w}{\sqrt{u-w^{2}}}\right) \arctan \left(\frac{w}{\sqrt{u-w^{2}}}\right)+\left(\tilde{k}_{2}-n\right) u \arctan \left(\frac{w}{\sqrt{u-w^{2}}}\right)^{2} \\
& +2\left(-b^{2}\left(\tilde{k}_{2}+\tilde{k}_{2}^{2}-2 \tilde{k}_{2} n+n(n-1)\right) w^{2}-\frac{\tilde{k}_{2} u v w}{\sqrt{u-w^{2}}}\right) \\
& \left.-\frac{1}{2}\left(\left(1+\tilde{a}+b^{2}\right) \tilde{k}_{2}-\left(\tilde{a}+b^{2}\right) n\right) u \log \left(w^{2}-u\right)\right) .
\end{aligned}
$$

Hence

$$
\begin{aligned}
F_{2}= & \alpha_{2} x^{2 \tilde{k}_{2}}\left(x^{2}+y^{2}\right)^{n-\tilde{k}_{2}-1}-\alpha_{0} x^{2 \tilde{k}_{2}-1}\left(x^{2}+y^{2}\right)^{n-\tilde{k}_{2}-2}\left(x\left(x^{2}+y^{2}\right)\left(\tilde{k}_{2}-n\right)\right. \\
& \arctan \frac{y}{x}\left(2 z+\arctan \frac{y}{x}\right)+2 b^{2}\left(\tilde{k}_{2}+\tilde{k}_{2}^{2}-2 \tilde{k}_{2} n+n(n-1)\right) y^{2} x \\
& \left.+x\left(x^{2}+y^{2}\right)\left(\tilde{k}_{2}+\left(\tilde{a}+b^{2}\right)\left(\tilde{k}_{2}-n\right)\right)(i \pi+2 \log x)+2 \tilde{k}_{2} y\left(x^{2}+y^{2}\right) z\right) .
\end{aligned}
$$

Taking into account that $F_{2}$ must be a weight-homogeneous polynomial of degree $2 n-2$ and that $\alpha_{0} \neq 0$ we must have that

$$
\tilde{k}_{2}-n=0 \quad \text { and } \quad \tilde{k}_{2}+\left(\tilde{a}+b^{2}\right)\left(\tilde{k}_{2}-n\right)=0,
$$


but this implies $\tilde{k}_{2}=n=0$ which is not possible because then $F_{0}$ would be a constant, implying that the invariant algebraic surface would be a constant. This concludes the proof of the theorem.

Proof of Theorem 3. In view of Theorem 5 if $f$ is an invariant algebraic surface of system (1) then it cannot be invariant by $\tau$. So, let $f$ be an invariant algebraic surface of system (1) which is not invariant by $\tau$. In view of Proposition 4, we get that $g=f \cdot \tau(f)$ is an invariant algebraic surface invariant by $\tau$, in contradiction with Theorem 5 . So, Theorem 3 is proved.

\section{ApPEndix}

5.1. Lyapunov coefficients. In this subsection, we present some basic notions about Hopf bifurcations and Lyapunov coefficients. The theory of Lyapunov coefficients can be found in [6, Chapters 3 and 10].

Consider the differential equation

$$
x^{\prime}=f(\mathbf{x}, \boldsymbol{\mu})
$$

where $\mathbf{x} \in \mathbb{R}^{n}$ and $\boldsymbol{\mu} \in \mathbb{R}^{m}$ are, respectively, vectors representing phase variables and control parameters.

A Hopf point $\left(\mathbf{x}_{0}, \boldsymbol{\mu}_{0}\right)$ is an equilibrium point of (12) where the Jacobian matrix $A=f_{\mathbf{x}}\left(\mathbf{x}_{0}, \boldsymbol{\mu}_{0}\right)$ has a pair of purely imaginary eigenvalues $\lambda_{ \pm}\left(\mu_{0}\right)=$ $\pm i \omega, \omega>0$ and admits no other eigenvalues with zero real part.

Denoting the variable $\mathbf{x}-\mathbf{x}_{0}$ also by $\mathbf{x}$ we write

$$
F(\mathbf{x})=f\left(\mathbf{x}, \boldsymbol{\mu}_{0}\right)
$$

as

$$
F(\mathbf{x})=A \mathbf{x}+\frac{1}{2} B(\mathbf{x}, \mathbf{x})+\frac{1}{6} C(\mathbf{x}, \mathbf{x}, \mathbf{x})+\mathcal{O}\left(\|\mathbf{x}\|^{4}\right)
$$

where $A=f_{\mathbf{x}}\left(0, \boldsymbol{\mu}_{0}\right)$,

$$
B_{i}(\mathbf{x}, \mathbf{y})=\left.\sum_{j, k=1}^{n} \frac{\partial^{2} F_{i}(\boldsymbol{\xi})}{\partial \xi_{j} \partial \xi_{k}}\right|_{\boldsymbol{\xi}=0} x_{j} y_{k}
$$

and

$$
C_{i}(\mathbf{x}, \mathbf{y}, \mathbf{z})=\left.\sum_{j, k, l=1}^{n} \frac{\partial^{3} F_{i}(\boldsymbol{\xi})}{\partial \xi_{j} \partial \xi_{k} \partial \xi_{l}}\right|_{\boldsymbol{\xi}=0} x_{j} y_{k} z_{l} .
$$

Let $p, q \in \mathbb{C}^{n}$ be vectors such that

$$
A q=i \omega q, \quad A^{\top} p=-i \omega p, \quad \bar{q} \cdot q=\bar{p} \cdot q=1,
$$


where $A^{\top}$ is the transposed of the matrix A. We define the first Lyapunov coefficient as

$$
\begin{aligned}
l_{1}= & \frac{1}{2 \omega} \operatorname{Re}\left(\bar{p} \cdot C(q, q, \bar{q})-2 \bar{p} \cdot B\left(q, A^{-1} \cdot B(q, \bar{q})\right)\right. \\
& \left.+\bar{p} B\left(\bar{q},\left(2 \omega i I_{n}-A\right)^{-1} B(q, q)\right)\right)
\end{aligned}
$$

where $I_{n}$ is the $n \times n$ identity matrix.

We have the following lemma from [6].

Lemma 6. Consider the differential system (12) having the Hopf point $\left(\mathbf{x}_{0}, \boldsymbol{\mu}_{0}\right)$ and assume that $l_{1} \neq 0$ and $\operatorname{Re}\left(\left.\frac{d \lambda_{ \pm}}{d \mu}\right|_{\mu=\mu_{0}}\right) \neq 0$. Then the following statements hold:

(i) If $l_{1}>0$, the differential system (12) has a supercritical Hopf bifurcation at $\mathbf{x}_{0}$.

(ii) If $l_{1}<0$, the differential system (12) has a subcritical Hopf bifurcation at $\mathbf{x}_{0}$.

5.2. Averaging theory. In this subsection we present recent results in averaging theory. For a general introduction to the averaging theory see the book of Sanders, Verhulst and Murdock [9].

We consider differential systems of the form

$$
\dot{\mathbf{x}}=\mathbf{F}_{0}(t, \mathbf{x})+\varepsilon \mathbf{F}_{1}(t, \mathbf{x})+\varepsilon^{2} \mathbf{F}_{2}(t, \mathbf{x})+\varepsilon^{3} \widetilde{\mathbf{F}}(t, \mathbf{x}, \varepsilon),
$$

with $\mathbf{x}$ in some open subset $\Omega$ of $\mathbb{R}^{n}, t \in[0, \infty), \varepsilon \in\left[-\varepsilon_{0}, \varepsilon_{0}\right]$. We assume $\mathbf{F}_{i}$ and $\widetilde{\mathbf{F}}$ for $i=1,2$ are $T$-periodic in the variable $t$. Let $\mathbf{x}(t, \mathbf{z}, 0)$ be the solution of the unperturbed system

$$
\dot{\mathbf{x}}=\mathbf{F}_{0}(t, \mathbf{x}),
$$

such that $\mathbf{x}(0, \mathbf{z}, 0)=\mathbf{z}$. We define $M(t, \mathbf{z})$ the fundamental matrix of the linear differential system

$$
\dot{\mathbf{y}}=\frac{\partial \mathbf{F}_{0}(t, \mathbf{x}(t, \mathbf{z}, 0))}{\partial \mathbf{x}} \mathbf{y}
$$

such that $M(0, \mathbf{z})$ is the identity. The displacement map of system (14) is defined as

$$
\mathbf{d}(\mathbf{z}, \varepsilon)=\mathbf{x}(T, \mathbf{z}, \varepsilon)-\mathbf{z}
$$

In order to have $\mathbf{d}(\mathbf{z}, \varepsilon)$ well defined we assume that for $|\varepsilon| \neq 0$ sufficiently small

$(H)$ there exists an open set $U \subset \Omega$ such that for all $\mathbf{z} \in U$ the unique solution $\mathbf{x}(t, \mathbf{z}, \varepsilon)$ is defined on the interval $\left[0, t_{(\mathbf{z}, \varepsilon)}\right)$ with $t_{(\mathbf{z}, \varepsilon)}>T$. 
The standard method of averaging for finding periodic solutions consists in writing the displacement map (15) as a power series of $\varepsilon$ in the following way

$$
\mathbf{d}(\mathbf{z}, \varepsilon)=\mathbf{g}_{0}(\mathbf{z})+\varepsilon \mathbf{g}_{1}(\mathbf{z})+\varepsilon^{2} \mathbf{g}_{2}(\mathbf{z})+\varepsilon^{3} \widetilde{\mathbf{g}}(\mathbf{z}, \varepsilon),
$$

where, for $i=0,1,2$, we have

$$
\mathbf{g}_{i}(\mathbf{z})=M(T, \mathbf{z})^{-1} \frac{\mathbf{y}_{i}(T, \mathbf{z})}{i !}
$$

being

$$
\begin{aligned}
\mathbf{y}_{0}(t, \mathbf{z})= & \mathbf{x}(t, \mathbf{z}, 0)-\mathbf{z} \\
\mathbf{y}_{1}(t, \mathbf{z})= & M(t, \mathbf{z}) \int_{0}^{t} M(\tau, \mathbf{z})^{-1} \mathbf{F}_{1}(\tau, \mathbf{x}(\tau, \mathbf{z}, 0)) \mathrm{d} \tau \\
\mathbf{y}_{2}(t, \mathbf{z})= & M(t, \mathbf{z}) \int_{0}^{t} M(\tau, \mathbf{z})^{-1}\left[2 \mathbf{F}_{2}(\tau, \mathbf{x}(\tau, \mathbf{z}, 0))\right. \\
(16) \quad & \left.+2 \frac{\partial \mathbf{F}_{1}}{\partial \mathbf{x}}(\tau, \mathbf{x}(\tau, \mathbf{x}, 0)) \mathbf{y}_{1}(\tau, \mathbf{z})+\frac{\partial^{2} \mathbf{F}_{0}}{\partial \mathbf{x}^{2}}(\tau, \mathbf{x}(\tau, \mathbf{z}, 0)) \mathbf{y}_{1}(\tau, \mathbf{z})^{2}\right] \mathrm{d} \tau .
\end{aligned}
$$

Let $\pi: \mathbb{R}^{m} \times \mathbb{R}^{n-m} \rightarrow \mathbb{R}^{m}$ and $\pi^{\perp}: \mathbb{R}^{m} \times \mathbb{R}^{n-m} \rightarrow \mathbb{R}^{n-m}$ denote the projections onto the first $m$ and $n-m$ coordinates, respectively. For a point $\mathbf{z} \in U$ we also consider $\mathbf{z}=(a, b) \in \mathbb{R}^{m} \times \mathbb{R}^{n-m}$. Take the graph

$$
\mathcal{Z}=\left\{\mathbf{z}_{\alpha}=(\alpha, \beta(\alpha)): \alpha \in \bar{V}\right\} \subset U
$$

such that $m<n, V$ is an open set of $\mathbb{R}^{m}$ and $\beta: \bar{V} \rightarrow \mathbb{R}^{n-m}$ is a $\mathbb{C}^{2}$ function.

The next theorem provides sufficient conditions for the existence of periodic solutions in the differential system (14).

Theorem 7 ([3, Theorem 1]). In addition to hypothesis $(H)$ assume that

(i) the averaged function $\mathbf{g}_{0}$ vanishes on the graph (17), that is, $\mathbf{g}_{0}\left(\mathbf{z}_{\alpha}\right)=$ 0 for all $\alpha \in \bar{V}$, and

(ii) the Jacobian matrix

$$
D \mathbf{g}_{0}\left(\mathbf{z}_{\alpha}\right)=\left(\begin{array}{cc}
\Lambda_{\alpha} & \Gamma_{\alpha} \\
B_{\alpha} & \Delta_{\alpha}
\end{array}\right)
$$

where

$$
\begin{aligned}
& \Lambda_{\alpha}=D_{a} \pi g_{0}\left(z_{\alpha}\right), \quad \Gamma_{\alpha}=D_{b} \pi g_{0}\left(z_{\alpha}\right), \\
& B_{\alpha}=D_{a} \pi^{\perp} g_{0}\left(z_{\alpha}\right), \quad \Delta_{\alpha}=D_{b} \pi^{\perp} g_{0}\left(z_{\alpha}\right) .
\end{aligned}
$$

satisfies that $\operatorname{det}\left(\Delta_{\alpha}\right) \neq 0$ for all $\alpha \in \bar{V}$. 
We define the functions

$$
\begin{aligned}
& f_{1}(\alpha)=-\Gamma_{\alpha} \Delta_{\alpha}^{-1} \pi^{\perp} \mathbf{g}_{1}\left(\mathbf{z}_{\alpha}\right)+\pi \mathbf{g}_{1}\left(\mathbf{z}_{\alpha}\right), \\
& f_{2}(\alpha)=\frac{1}{2} \Gamma_{\alpha} \gamma_{2}(\alpha)+\frac{1}{2} \frac{\partial^{2} \pi \mathbf{g}_{0}}{\partial b^{2}}\left(\mathbf{z}_{\alpha}\right) \gamma_{1}(\alpha)^{2}+\frac{\partial \pi \mathbf{g}_{1}}{\partial b}\left(z_{\alpha}\right) \gamma_{1}(\alpha)+\pi \mathbf{g}_{2}\left(\mathbf{z}_{\alpha}\right), \\
& \gamma_{1}(\alpha)=-\Delta_{\alpha}^{-1} \pi^{\perp} \mathbf{g}_{1}\left(\mathbf{z}_{\alpha}\right), \\
& \gamma_{2}(\alpha)=-\Delta_{\alpha}^{-1}\left(\frac{\partial^{2} \pi^{\perp} \mathbf{g}_{0}}{\partial b^{2}}\left(\mathbf{z}_{\alpha}\right) \gamma_{1}(\alpha)^{2}+2 \frac{\partial \pi^{\perp} \mathbf{g}_{1}}{\partial b}\left(\mathbf{z}_{\alpha}\right) \gamma_{1}(\alpha)+2 \pi^{\perp} \mathbf{g}_{2}(\alpha)\right) .
\end{aligned}
$$

Then the following statements hold.

(a) If there exists $\alpha^{*} \in V$ such that $f_{1}\left(\alpha^{*}\right)=0$ and $\operatorname{det}\left(D f_{1}\left(\alpha^{*}\right)\right) \neq 0$, for $|\varepsilon| \neq 0$ sufficiently small, then there is an initial condition $\mathbf{z}(\varepsilon) \in$ $U$ such that $\mathbf{z}(0)=\mathbf{z}_{\alpha^{*}}$ and the solution $\mathbf{x}(t, \mathbf{z}(\varepsilon), \varepsilon)$ of system (14) is $T$-periodic.

(b) Assume that $f_{1} \equiv 0$. If there exists $\alpha^{*} \in V$ such that $f_{2}\left(\alpha^{*}\right)=0$ and $\operatorname{det}\left(D f_{2}\left(\alpha^{*}\right)\right) \neq 0$, then for $|\varepsilon| \neq 0$ sufficiently small, there is an initial condition $\mathbf{z}(\varepsilon) \in U$ such that $\mathbf{z}(0)=\mathbf{z}_{\alpha^{*}}$ and the solution $\mathbf{x}(t, \mathbf{z}(\varepsilon), \varepsilon)$ of system (14) is T-periodic.

\section{ACKNOWLEDGEMENTS}

The first author is supported by CNPq 248501/2013-5. The second author is supported by the Ministerio de Economía, Industria y Competitividad, Agencia Estatal de Investigacin grant MTM2016-77278-P (FEDER), the Agència de Gestió d'Ajuts Universitaris i de Recerca grant 2017 SGR 1617, and the European project Dynamics-H2020-MSCA-RISE-2017-777911. The third author is partially supported by FCT/Portugal through UID/MAT/ $04459 / 2013$. The third author is partially supported by is partially supported by Fondecyt project 1130644 .

\section{REFERENCES}

[1] A. Arikoglu And I. Ozkol, Solution of fractional integro-differential equations by using fractional differential transform method, Chaos, Solitons \& Fractals 40 (2009), 521-529.

[2] M.K. Bouafoura, O. Moussi, O And N.B. Braiek, A fractional state space realization method with block pulse basis, Signal. Process. 91 (2011), 492-497.

[3] M. R. CÂNDIDO AND J. LlibRE, Stability of periodic orbits in the averaging theory: Applications to Lorenz and Thomas' differential systems, Inter. J. Bifurcation and Chaos 28 (2018) 1830007, pp. 14.

[4] A. Deb, A. Dasgupta And G. Sarkar, A new set of orthogonal functions and its application to the analysis of dynamic systems, J. Frankl. Inst. 343 (2006), 1-26.

[5] H.R. Fazli, F. Hassani, A. Ebadian and A.A. Khajehnasiri, National economies in state-space of fractional-order financial system, Afr. Mat. 27 (2016), 529-540.

[6] Y. A. Kuznetsov, Elements of applied bifurcation theory, 112, Springer Science \& Business Media, 2013. 
[7] J. Llibre, C. Stoica and C. Valls, Polynomial and rational integrability of polynomial Hamiltonian systems, Electron. J. Differential Equations, 2012 (2012), 1-6.

[8] S. Momani And M.A. Noor, Numerical methods for fourth-order fractional integrodifferential equations, Appl. Math. Comput. 182 (2006), 754-760.

[9] J.A. Sanders, F. Verhulst And J. Murdock, Averaging methods in nonlinear dynamical systems, Second edition. Applied Mathematical Sciences 59. Springer, New York, 2007.

[10] T. Skovrnek, I., Podlubny and I. Petrs, Modeling of the national economies in state-space: a fractional calculus approach, Econ. Model. 29 (2012), 1322-1327.

${ }^{1}$ Departament de Matemàtiques, Universitat Autònoma de Barcelona, 08193 Bellaterra, Barcelona, Catalonia, Spain

E-mail address: candidomr@mat.uab.cat

E-mail address: jllibre@mat.uab.cat

2 Departamento de Matemática, Instituto Superior Técnico, Universidade De Lisboa, Av. Rovisco Pais 1049-001, Lisboa, Portugal

E-mail address: cvalls@math.ist.utl.pt 